\title{
PEGylated Gold Nanoparticles with Interesting Plasmonic Properties Synthesized Using an Original, Rapid, and Easy-to-Implement Procedure
}

\author{
Ștefan Nițică, ${ }^{1}$ Alin Iulian Moldovan, ${ }^{1}$ Valentin Toma, ${ }^{1}$ Cristian Silviu Moldovan, \\ Ioana Berindan-Neagoe $\mathbb{D}^{1},{ }^{1,2,3}$ Gabriela Știufiuc $\mathbb{D}^{4},{ }^{4}$ Constantin M. Lucaciu $\mathbb{D}^{5}{ }^{5}$ \\ and Rareș Știufiuc $\mathbb{D}^{1,5}$

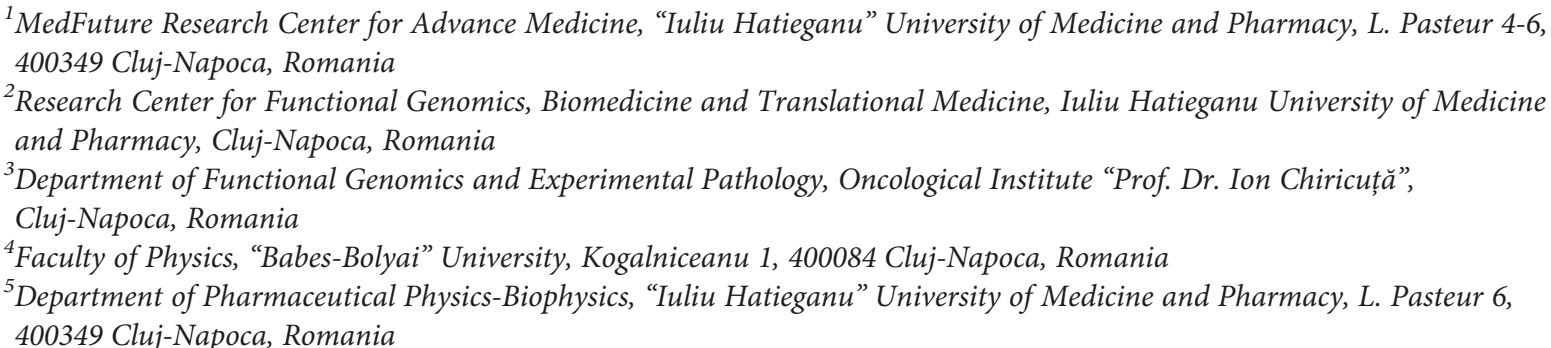

Correspondence should be addressed to Gabriela Știufiuc; gabi.stiufiuc@phys.ubbcluj.ro

Received 10 July 2018; Revised 31 August 2018; Accepted 11 September 2018; Published 11 October 2018

Academic Editor: Ilaria Fratoddi

Copyright (C) 2018 Ștefan Nițică et al. This is an open access article distributed under the Creative Commons Attribution License, which permits unrestricted use, distribution, and reproduction in any medium, provided the original work is properly cited.

\begin{abstract}
In this letter, we report a new, one-step, rapid, and easy-to-implement method for the synthesis of PEGylated gold nanoparticles (PEG-AuNPs) having a narrow size distribution and very interesting plasmonic properties. Unmodified polyethylene glycol molecules with a molecular weight of $1000 \mathrm{~g} / \mathrm{mole}$ (PEG1000) have been employed as reducing and capping agents for the synthesis of spherical gold nanoparticles having an average diameter of $35 \mathrm{~nm}$, within a few minutes. The novelty of the herein proposed synthesis method consists in the fact that the synthesis takes place inside of a sealed bottle flask containing aqueous solutions of PEG1000, tetrachloroauric(III) acid $\left(\mathrm{HAuCl}_{4}\right)$, and $\mathrm{NaOH}$, placed in the center of a microwave oven, capable to provide a very uniform temperature environment. It turned out that, during the very short synthesis procedure ( 2 minutes), PEG 1000 suffers an oxidative transformation in such a manner that its terminal alcohol groups $\left(-\mathrm{CH}_{2}-\mathrm{OH}\right)$ are transformed in carboxylate ones $\left(-\mathrm{COO}^{-}\right)$. The as-synthesized PEG-AuNPs possess very interesting plasmonic properties allowing the detection of different molecules by means of SER spectroscopy performed either in liquid droplets or on solid spots. As a consequence of their unique plasmonic properties, the SER spectra acquired using this new class of nanoparticles on different molecules of interest (methylene blue, rhodamine 6G, doxorubicin, and 5-fluorouracil) are highly reproducible, making them ideal candidates for further use as SERS substrates.
\end{abstract}

\section{Introduction}

Although they were used since ancient times for decorative and medical purposes [1], gold colloids became intensively studied only in the last decades [2]. Their nowadays popularity in the field of nanotechnology resides in the fact that gold nanoparticles possess unique optoelectronic characteristics related to the occurrence of a surface plasmon resonance (SPR) band $[2,3]$ as their size decreases to the nanoscale. Moreover, these properties are tunable [4], making them interesting candidates for biomedical applications [3-5] such as SERS-based detection [6, 7], plasmonic photothermal therapy $[5,7,8]$, and drug and gene delivery applications $[5,7]$. 
A major drawback of these nanoparticles is their relative instability in biological fluids due to the interaction with biomolecules such as opsonins that further promote their uptake by mononuclear phagocyte system (MPS) cells [3, 9] if used in in vivo experiments. The unspecific attachment of these large molecules on the nanoparticles also prevents other molecules to reach the critical distance from the metallic surface, a sine quo non condition for the surface enhanced Raman scattering (SERS) phenomenon to take place. Consecutively, the use of undecorated gold nanoparticles as SERS substrates may be hampered when studying biological fluids. A strategy to overcome these challenges is to attach polyethylene glycol (PEG) to their surface $[9,10]$ in order to form a thin hydrated barrier that sterically prevents the opsonization process [10]. This way, the blood half-life of AuNPs substantially increases and the probability of reaching their target (malignant or inflamed tissue) by enhanced permeability and retention (EPR) effect is also increased [9]. The AuNPs PEGylation could further promote their use as SERS substrates, especially if the PEG layer is thin enough to permit other molecules to reach an optimum distance from their surface. Moreover, if properly understood, the interaction of the PEG molecules with the analytes could have major implications in terms of improving spectral reproducibility for ultrasensitive biodetection applications.

The most commonly used approach to PEGylate the gold nanoparticles is to introduce thiol-modified PEG molecules in a preformed gold colloid $[5,7,9,11]$. Thus, PEG attaches to the gold surface by a place-exchange reaction, displacing the initial capping ligands due to the higher affinity of the sulfhydryl group for gold [5, 7]. As a consequence, in order to obtain PEGylated gold nanoparticles, a two-step process involving the synthesis of gold nanoparticles followed by the attachment of thiol-modified PEG molecules is required in the vast majority of the methods published in the scientific literature.

Over the years, our research group has developed new strategies for the synthesis of PEGylated plasmonic (gold and silver) nanoparticles with great Raman enhancement factors [4, 12]. Here, we report a new, quick, one-step microwave-assisted synthesis method of monodisperse spherical PEGylated gold nanoparticles covered with a uniform molecular layer of PEG1000. The novelty of the here reported synthesis method, compared to other approaches developed for the fabrication of PEGylated AuNPs [13], consists in the fact it uses only unmodified PEG1000 as reducing, capping, and stabilizing agent. The as-obtained spherical gold nanoparticles have a good monodispersivity and proved to be excellent plasmonic substrates, allowing the reproducible detection of several analytes (methylene blue, rhodamine 6G, doxorubicin, and 5-fluorouracil) in liquid droplet or on solid spots by means of SER spectroscopy.

\section{Materials and Methods}

2.1. Materials. Tetrachloroauric(III) acid and trisodium citrate dihydrate were purchased from Sigma-Aldrich (Germany). Polyethylene glycol with a molecular weight of $1000 \mathrm{~g} / \mathrm{mol}$ (designated PEG 1000) was purchased from
Carl Roth (Germany) and used without further purification. Sodium hydroxide was provided by Lach-Ner (Czech Republic), and ultrapurified water (18.2 $\mathrm{m} \Omega$, Chorus Purelab, Elga) was used as a solvent. $\mathrm{CaF}_{2}$-polished glass acting as port probes in SERS experiments has been purchased from Crystran Limited UK.

2.2. Preparation of Gold Nanoparticles. The synthesis of PEGylated gold nanoparticles (PEG-AuNPs) was performed by uniformly heating an aqueous solution containing PEG 1000, $\mathrm{H}\left[\mathrm{AuCl}_{4}\right]$, and $\mathrm{NaOH}$ placed inside of a sealed bottle flask. Briefly, $1 \mathrm{~g}$ of PEG $1000,550 \mu \mathrm{l}$ of a $128 \mathrm{mM}$ aqueous solution of $\mathrm{H}\left[\mathrm{AuCl}_{4}\right]$, and $1.5 \mathrm{ml}$ of $\mathrm{NaOH} 1 \%(\mathrm{M} / \mathrm{V})$ were added to $48 \mathrm{ml}$ of ultrapurified water in a Duran pressureresistant flask and heated for $2 \mathrm{~min}$ in a microwave oven (Sharp R-25AM 2100W). After 90 seconds of heating, the solution color became ruby red, indicating the successful synthesis of the gold colloid.

2.3. Synthesis of Solid SERS PEG-AuNPs Substrate. The solid SERS PEG-AuNPs substrates were synthesized by pouring a small amount $(1 \mu \mathrm{l})$ of a mixture of PEG-AuNPs with the analytes on a $\mathrm{CaF}_{2}$-polished glass, acting as a "port probe" substrate. The analyte: PEG-AuNPs mixtures have been realized at a $1: 1$ rate, for 5 minutes at room temperature. The port probe temperature has been set to $50^{\circ} \mathrm{C}$ before the deposition of the mixture. The drying time of the mixture was set to $90 \mathrm{~s}$. During this period of time, the mixture self-assembled as micrometric PEG-AuNPs-aggregated islands. The solid SERS substrates have been left for 15 minutes at room temperature prior to SERS measurements. The SERS spectra have been acquired at room temperature from different regions of the PEG-AuNPs islands. Each presented spectrum represents the mean of 625 individual spectra recorded on different regions of the solid substrate.

2.4. Methods and Instrumentation. The VIS absorption spectra were recorded using a T92+ UV-VIS spectrophotometer from PG Instruments. The absorption curves have been acquired on standard plastic cells at room temperature, over a spectral range between $400 \mathrm{~nm}$ and $800 \mathrm{~nm}$. The spectral resolution has been set at $2 \mathrm{~nm}$.

Electron microscopy measurements were performed on a HT7700 (Hitachi, Japan) transmission electron microscope (TEM) operating at $120 \mathrm{kV}$, using the high-resolution operation mode.

The dynamic light scattering (DLS) analysis has been performed on a $\mathrm{Vasco}^{\gamma}$ nanoparticle analyzer (Cordouan Technologies, France) using a monochromatic laser beam having a wavelength of $658 \mathrm{~nm}$ and $65 \mathrm{~mW}$ power. Zeta potential measurements were taken using a Wallis ${ }^{\zeta}$ zeta potential analyzer (Cordouan Technologies, France).

FT-IR spectra were recorded on samples of dried PEGylated gold colloid and pure PEG 1000 molecules. In order to remove the free PEG from the solution, the PEGylated gold colloid sample was prepared by centrifuging a small volume of colloid $(2 \mathrm{ml})$ at $5000 \mathrm{rpm}$ for $10 \mathrm{~min}$, followed by the removal of the supernatant and by the redispersion of the sediment in an equal volume of ultrapurified water. In a final 
step, the washed colloid was centrifuged one more time using the same parameters. After the removal of the supernatant, a small droplet of the sediment was placed on the crystal of a TENSOR II FT-IR spectrometer (Bruker, Germany) and left to dry.

The SERS measurements performed on liquid solutions have been recorded in $1 \mathrm{ml}$ glass vials filled with $540 \mu \mathrm{l}$ of colloid and $60 \mu \mathrm{l}$ of analyte (methylene blue solutions of multiple concentrations) in the $200-2000 \mathrm{~cm}^{-1}$ range, using a DeltaNu Advantage spectrometer (DeltaNu, Laramie, WY) equipped with a laser diode emitting at $785 \mathrm{~nm}$. The laser power was $100 \mathrm{~mW}$, and the spectral resolution was $5 \mathrm{~cm}^{-1}$. Each SERS spectrum is the average of 10 recordings taken with an acquisition time of 10 seconds.

The SERS measurements performed on solid SERS substrate (PEG-AuNPs islands) have been recorded using a confocal Renishaw ${ }^{\circledR}$ inVia microscope (Renishaw plc, Wotton-under-Edge, Gloucestershire, UK), equipped with a Leica microscope (Leica Microsystems GmbH, Germany), using a 50x objective (N.A. 0.75) and a 100x objective (N.A. 0.85), respectively. A $785 \mathrm{~nm}$ diode laser (Renishaw, UK) has been used for excitation. The laser power (measured at the substrate surface) was less than $6.3 \mathrm{~mW}$, and the acquisition time was set to $10 \mathrm{~s}$. The spectrograph was equipped with a 1200 lines/mm grating and a charge coupled device camera (CCD).

\section{Results and Discussions}

In our experiments, we have observed that the PEG-mediated reduction of tetrachloroauric(III) acid is significantly sped up and the resulting gold colloids are highly monodisperse and stable if the reaction media is alkaline. Thus, we have decided to add sodium hydroxide $(\mathrm{NaOH})$ to the reaction mixture in order to elevate its $\mathrm{pH}$ to a slightly alkaline value $(\sim 9)$.

Before introducing the reaction mixture in the microwave oven, it has been observed that the pale yellow color of the diluted tetrachloroauric(III) acid solution has instantaneously shifted to colorless after pouring the $\mathrm{NaOH}$ solution. According to previous studies [14-16], this is a proof that a ligand-exchange reaction, involving the tetrachloroaurate (III) ion, took place. Briefly, one or more chloride ions from the tetrachloroaurate complex were displaced by hydroxide ions according to the following reaction:

$\left[\mathrm{AuCl}_{4}\right]+\mathrm{nOH}^{-} \rightarrow\left[\mathrm{AuCl}_{4-\mathrm{n}}(\mathrm{OH})_{\mathrm{n}}\right]+\mathrm{nCl}^{-}$(adaptation from [15]), leading to a decrease in the intensity of the ligand-to-metal charge transfer transition phenomenon and consecutively to the loss of the yellow color of the complex due to a significant blue shift in the absorption spectra [14-16]. Given the facts that hydroxy-gold(III) derivatives have greater reduction potentials compared to tetrachloroaurate(III) ions [17] and the excess hydroxide ions could promote the deprotonation of several reaction intermediates with a consecutive chemical equilibrium shift towards the formation of oxidation products, we can conclude that alkaline reaction environment induces the oxidation of relatively stable PEG 1000 molecule. As a further proof of this conclusion, we have observed that the synthesis of PEGylated AuNPs can be obtained only after the addition of $\mathrm{NaOH}$ in the reaction mixture. Moreover, in the absence of $\mathrm{PEG}$, the reduction of $\mathrm{H}\left[\mathrm{AuCl}_{4}\right]$ does not take place.

The TEM images of the as-synthesized gold colloids revealed the presence of well-defined spherical gold nanoparticles (Figure 1(a)) confirming that the here proposed synthesis method can generate in a very short period of time the spherical gold nanoparticles (AuNPs) coated with a uniform shell of PEG1000 molecules having a 3-5 nm thickness (Figure 1(b)). The statistical analysis of the TEM images illustrated that AuNPs have a narrow size distribution with an average size of $35 \mathrm{~nm}$ (Figure 1(c)). As it was expected from the visual examination of the gold colloid (having an intense ruby-red color), its VIS absorption spectrum exhibited a well-defined peak at $530 \mathrm{~nm}$, corresponding to the surface plasmon resonance band (Figure 1(d)).

The DLS measurements (Figure 2) revealed a mean hydrodynamic diameter of circa $39 \mathrm{~nm}$, which is in good agreement with the value obtained from TEM measurements. The small difference is given by the presence of the surface coating on the outer surface of PEG-AuNPs, poorly visible but still distinguishable in the TEM image of an individual nanoparticle (Figure 1(b)). The presence of a surface coating was also confirmed by zeta potential measurements, as the obtained results indicated that the PEGylated AuNPs had a negative zeta potential with a mean value of $-31.25 \mathrm{mV}$. If one considers the threshold value of stability at $\pm 25 \mathrm{mV}$, it can be stated that the as-synthesized gold colloid possesses a good electrostatic repulsion driven stability [5].

Further investigations on the nature of the PEG-AuNPs' surface coating by FT-IR spectroscopy revealed that AuNPs had PEG1000 molecules attached to their surface, as their FT-IR spectrum presented the same vibrational peaks as the FT-IR spectrum of pure PEG1000 (Figure 3). A very interesting aspect that needs to be outlined is the presence of $1656 \mathrm{~cm}^{-1}$ vibrational peak in the FT-IR spectrum of PEGAuNPs. This vibrational peak is assigned to an asymmetric stretching vibrational mode of gold carboxylate unidentate bond [6]. This observation leads to the idea that PEG1000 molecules suffer an oxidative transformation during the formation of the gold nanoparticles in such a manner that the terminal alcohol groups $\left(-\mathrm{CH}_{2}-\mathrm{OH}\right)$ are transformed in carboxylate ones $\left(-\mathrm{COO}^{-}\right)$. This can explain the interaction of PEG molecules with gold nanoparticles and the existence of a negative charge on their surface. Thus, one can conclude that PEG1000 serves as reducing, capping, and stabilizing agent for AuNPs in the here proposed synthesis method.

The plasmonic properties of PEG-AuNPs were firstly tested by recording SER spectra of methylene blue (MB) analyte in aqueous solutions, using these NPs as substrates. Figure 4 shows the SER spectra of $\mathrm{MB}$ at molar concentrations comprised between $10^{-3} \mathrm{M}$ and $10^{-7} \mathrm{M}$. The characteristic peaks of $\mathrm{MB}$ could be observed down to a concentration of $10^{-6} \mathrm{M}$. It can be observed that at a concentration of $10^{-4} \mathrm{M}$, the SER spectrum of $\mathrm{MB}$ is very intense and slightly different from those acquired at other analyte concentrations.

The major vibrational peaks of $\mathrm{MB}$ are presented in all spectra, indicating a similar adsorption geometry of the 


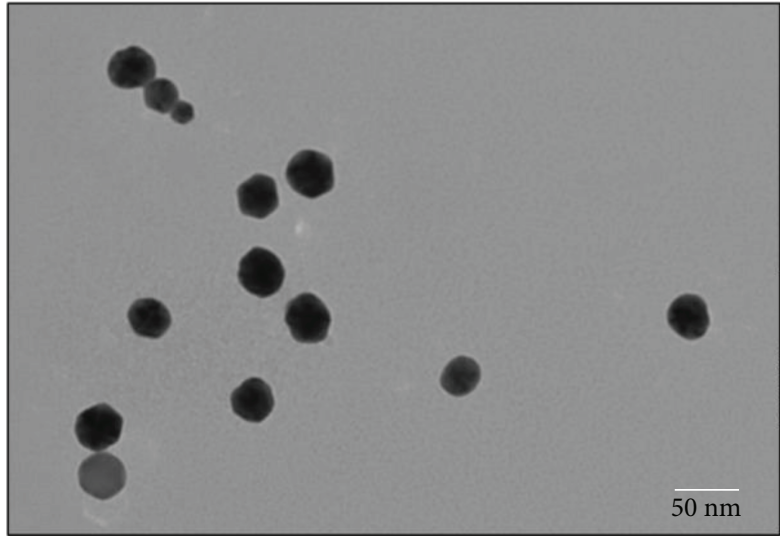

(a)

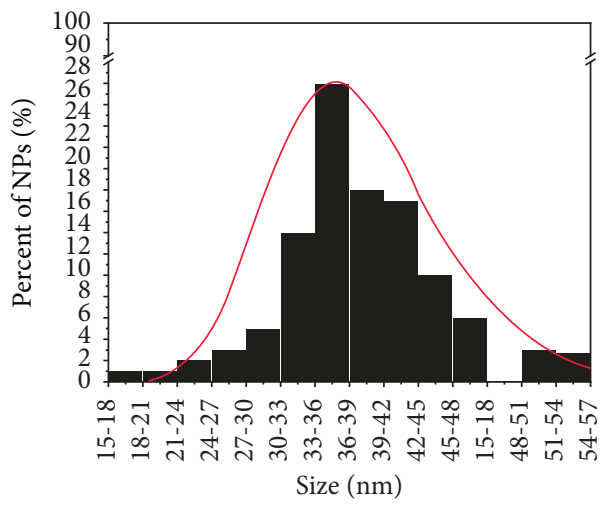

AuPEG 1000 NPs

(c)

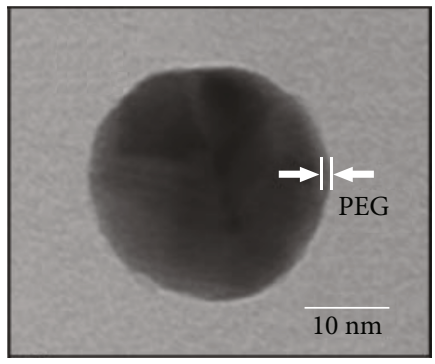

(b)

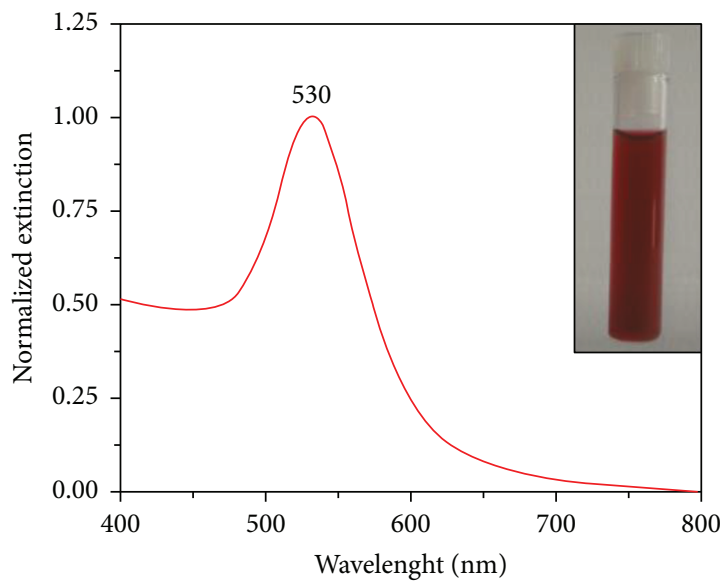

(d)

FIGURE 1: TEM image of gold colloids (a). TEM image of an individual PEGylated gold nanoparticle (b). Size distribution of gold nanoparticles obtained from statistical analysis of TEM images (c). UV-VIS absorption spectrum of the gold colloid (d) optical image of the gold colloid solution (inset d).

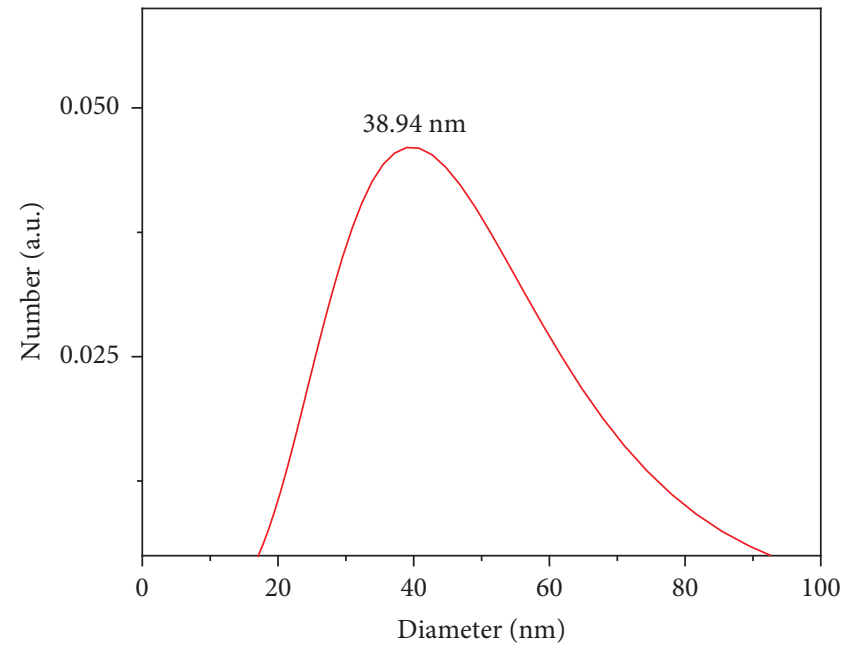

(a)

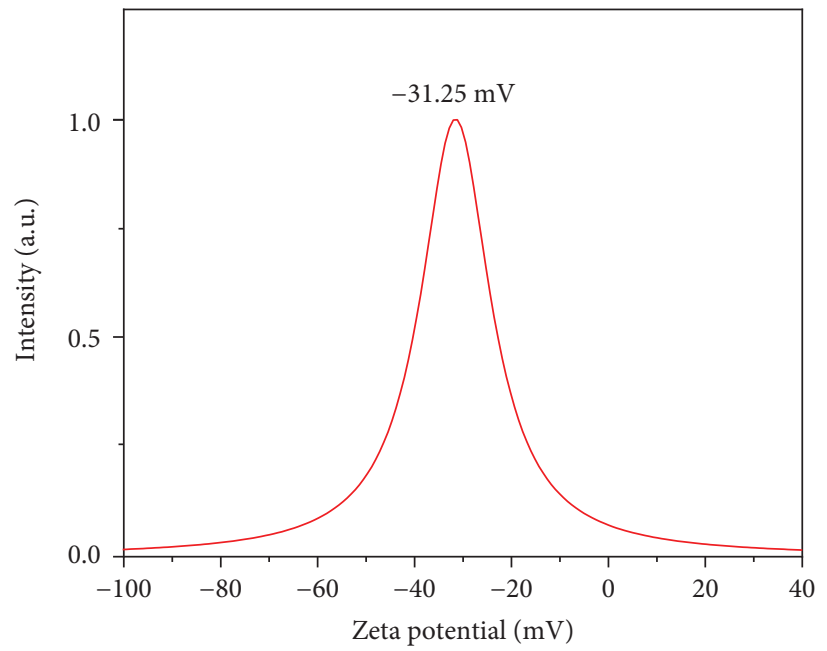

(b)

Figure 2: (a) DLS results (cumulants) of PEG-AuNPs colloids. (b) Zeta potential measurements of PEG-AuNPs colloids. 


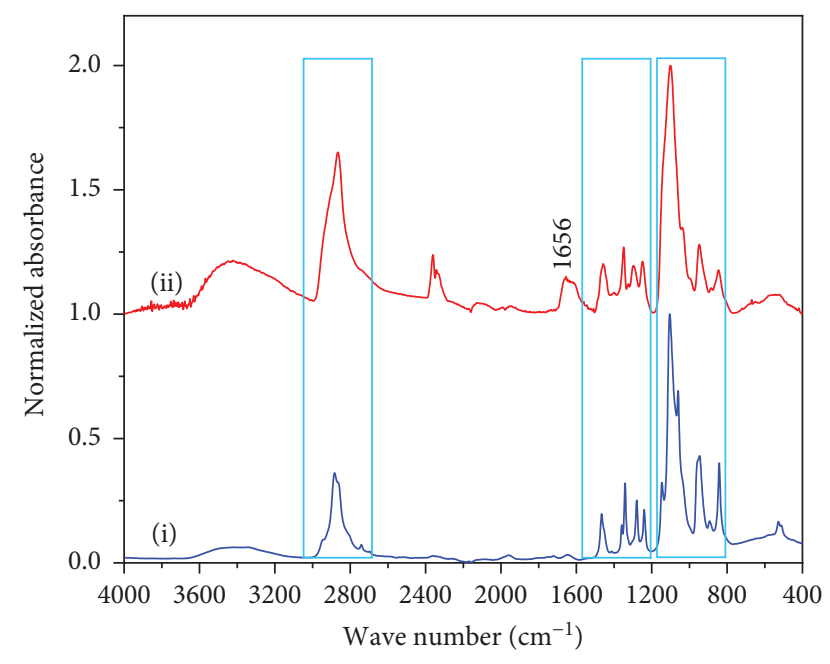

FIgURE 3: FT-IR spectra of pure PEG1000 molecules (i) and of PEG-AuNPs (ii).

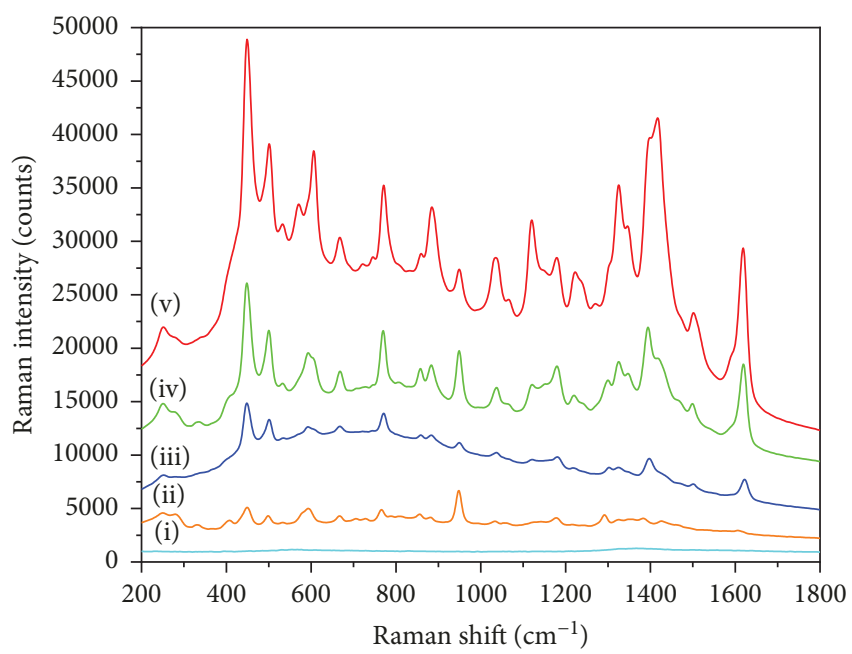

FIGURE 4: SER spectra of MB at different molar concentrations, acquired using PEG-AuNPs as substrate: (i) $10^{-7} \mathrm{M}$, (ii) $10^{-6} \mathrm{M}$, (iii) $10^{-3} \mathrm{M}$, (iv) $10^{-5} \mathrm{M}$, and (v) $10^{-4} \mathrm{M}$.

analyte with respect to nanoparticle surface in the whole analyte concentration range.

Figure 5 reveals the hyperspectral image of PEG-AuNPs (Figure 5(a)) and the mean reflectance spectra of the most commonly observed classes of PEG-AuNPs, in the field of view (Figure 5(b)). The nanoparticles appear bright as a consequence of their high scattering cross section, thus allowing the optical detection of small nanoparticles clusters or even individual ones. It has been recently shown that gold nanoparticles have unique reflectance spectra presenting well-defined peaks located between 550 and $650 \mathrm{~nm}$ [18]. By examining the reflectance spectra acquired on the most dominant three classes of nanoparticles detected in the field of view (green, red, and orange), one can observe that each of these spectra presents a well-defined peak located between 550 and $650 \mathrm{~nm}$ (Figure 5(b)). Hyperspectral microscopy provided a further proof of the successful synthesis of PEG-AuNPs.

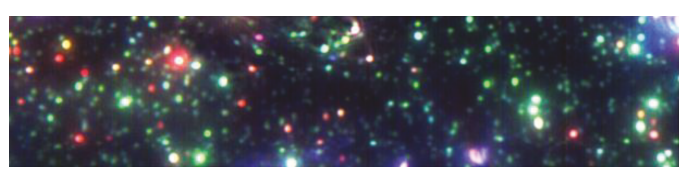

(a)

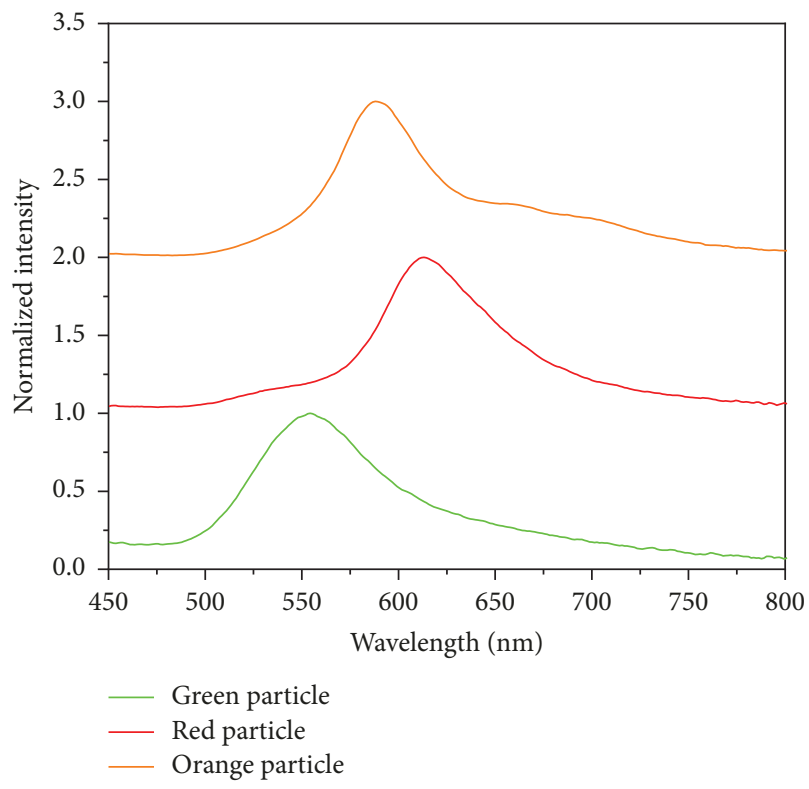

(b)

FIgUre 5: Hyperspectral image of PEG-AuNPs (a). The mean reflectance spectra of the three most commonly observed classes of PEG-AuNPs (b).

Given their plasmonic properties, ease of fabrication, and stability, one of the most promising applications of the here proposed PEG-AuNPs could be their use as main building blocks for the production of solid plasmonic substrates for the detection of different pharmaceutical compounds using ultrasensitive vibrational spectroscopy (SERS). Figure 6 presents the SER spectra of a test molecule (rhodamine 6G-R6G) and of two antineoplastic compounds (5-fluorouracil-5FU and doxorubicin-DOX) recorded using solid substrates produced from PEG-AuNPs. As it can be seen on the figures, each solid substrate is capable to generate well-resolved high-quality spectra for each tested compound. Moreover, a high reproducibility of the recorded spectra and a very small intensity variation have been detected with respect to the position on the substrate where the spectra have been recorded. This observation could represent a major finding in the field of SERS-based biodetection applications if one considers that one of the major obstacles that hampers SERS implementation in clinical practice is represented by its lack of reproducibility.

\section{Conclusions}

In this work, we have successfully synthesized, characterized, and tested in terms of plasmonic properties the PEGylated gold nanoparticles using a simple, one-step, microwaveassisted procedure. The obtained gold nanoparticles were 


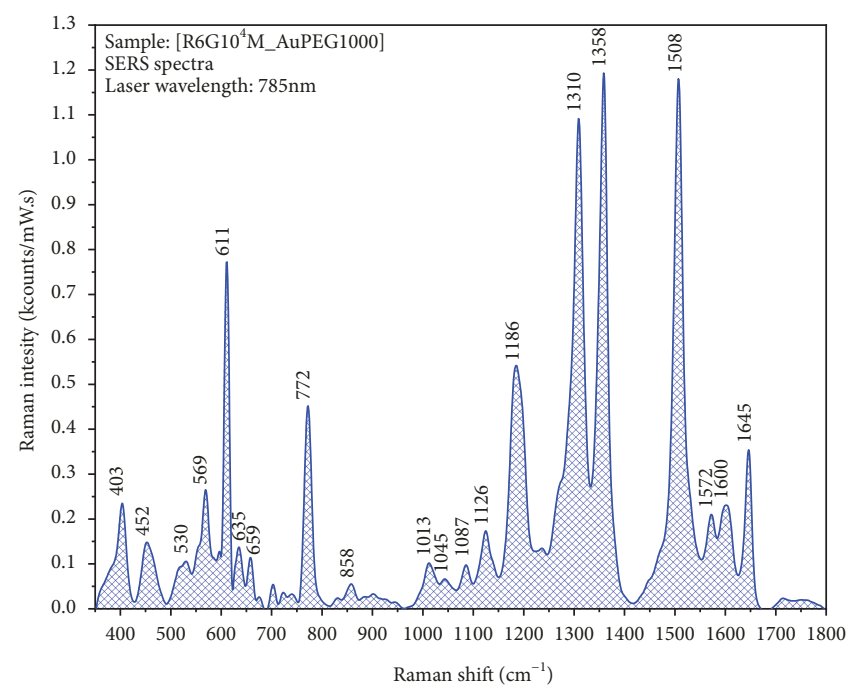

(a)

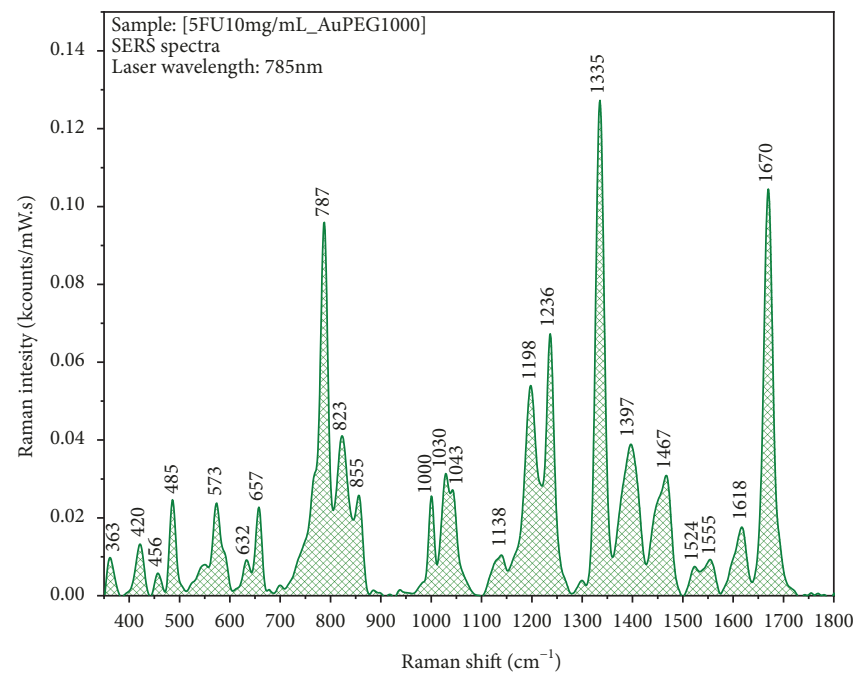

(b)

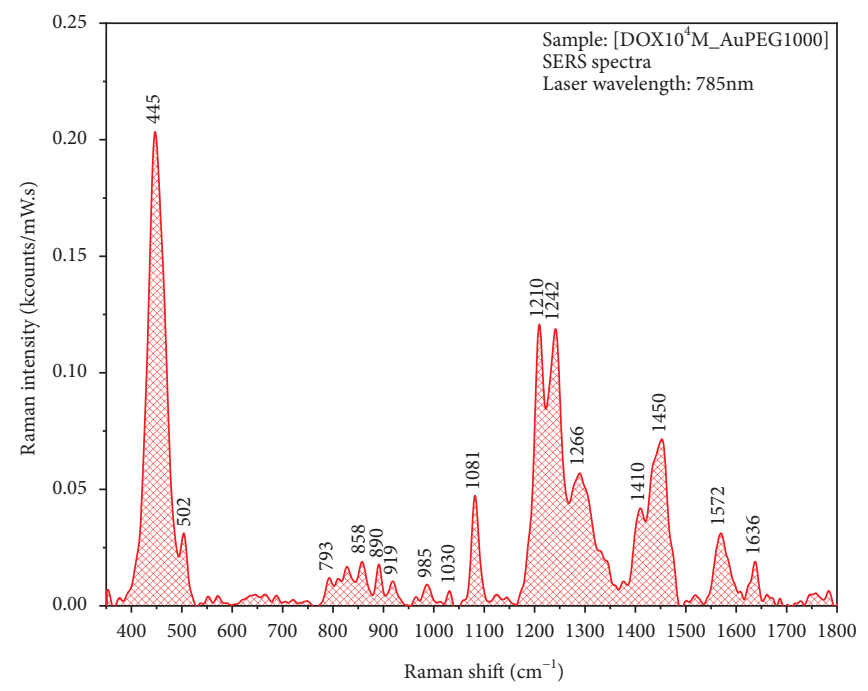

(c)

FIGURE 6: SER spectra of R6G (a), 5-FU (b), and DOX (c) acquired on solid substrates produced from as-synthesized PEG-AuNPs, using an excitation wavelength of $785 \mathrm{~nm}$. The analyte concentration in each case is $10^{-3} \mathrm{M}$. Each spectrum is the mean of 625 individual spectra, acquired on different regions of the substrate.

highly monodisperse, having a mean diameter of $35 \mathrm{~nm}$. PEG molecules were shown to have multiple roles in the formation and stabilization of the gold nanoparticles. It has been shown that an alkaline environment is a critical parameter for a successful synthesis and that PEG molecules act both as reducing and capping agent, as shown by the FT-IR measurements acquired on gold colloid. Moreover, DLS and zeta potential measurements have additionally proven that oxidized PEG molecules are adsorbed on the surface of the AuNPs, while the PEG layer can be faintly observed in the TEM images of the gold nanoparticles. SERS measurements have shown that the as-obtained gold colloid can be successfully used as plasmonic substrate in order to acquire high-quality spectra of several analytes in a very reproducible manner in liquid or solid conditions.

\section{Data Availability}

All experimental data (TEM images, SERS spectra, DLS data, and FT-IR spectra) used to support the findings of this study are available from the corresponding author upon request.

\section{Conflicts of Interest}

The authors declare that they have no conflicts of interest.

\section{Acknowledgments}

Our group highly acknowledges the financial support from the Competitiveness Operational Programme 2014-2020 POC-A1-A1.1.4-E-2015, financed under the European Regional Development Fund, project number P_37_765. 


\section{References}

[1] M. C. Daniel and D. Astruc, "Gold nanoparticles: assembly, supramolecular chemistry, quantum-size-related properties, and applications toward biology, catalysis, and nanotechnology," Chemical Reviews, vol. 104, no. 1, pp. 293-346, 2004.

[2] S. Eustis and M. A. El-Sayed, "Why gold nanoparticles are more precious than pretty gold: noble metal surface plasmon resonance and its enhancement of the radiative and nonradiative properties of nanocrystals of different shapes," Chemical Society Reviews, vol. 35, no. 3, pp. 209-217, 2006.

[3] K. Rahme, L. Chen, R. G. Hobbs, M. A. Morris, C. O'Driscoll, and J. D. Holmes, "PEGylated gold nanoparticles: polymer quantification as a function of PEG lengths and nanoparticle dimensions," RSC Advances, vol. 3, no. 17, pp. 6085-6094, 2013.

[4] R. Stiufiuc, C. Iacovita, R. Nicoara et al., "One-step synthesis of PEGylated gold nanoparticles with tunable surface charge," Journal of Nanomaterials, vol. 2013, Article ID 146031, 7 pages, 2013.

[5] P. Ghosh, G. Han, M. De, C. Kim, and V. Rotello, "Gold nanoparticles in delivery applications," Advanced Drug Delivery Reviews, vol. 60, no. 11, pp. 1307-1315, 2008.

[6] S. S. Agasti, S. Rana, M.-H. Park, C. K. Kim, C.-C. You, and V. M. Rotello, "Nanoparticles for detection and diagnosis," Advanced Drug Delivery Reviews, vol. 62, no. 3, pp. 316-328, 2010.

[7] R. A. Sperling, P. Rivera Gil, F. Zhang, M. Zanella, and W. J. Parak, "Biological applications of gold nanoparticles," Chemical Society Reviews, vol. 37, no. 9, pp. 1896-1908, 2008.

[8] X. Huang, P. K. Jain, I. H. El-Sayed, and M. A. El-Sayed, "Plasmonic photothermal therapy (PPTT) using gold nanoparticles," Lasers in Medical Science, vol. 23, no. 3, pp. 217-228, 2008.

[9] J. V. Jokerst, T. Lobovkina, R. N. Zare, and S. S. Gambhir, "Nanoparticle PEGylation for imaging and therapy," Nanomedicine, vol. 6, no. 4, pp. 715-728, 2011.

[10] J. S. Suk, Q. Xu, N. Kim, J. Hanes, and L. M. Ensign, "PEGylation as a strategy for improving nanoparticle-based drug and gene delivery," Advanced Drug Delivery Reviews, vol. 99, no. Part A, pp. 28-51, 2016.

[11] E. C. Dreaden, A. M. Alkilany, X. Huang, C. J. Murphy, and M. A. El-Sayed, "The golden age: gold nanoparticles for biomedicine," Chemical Society Reviews, vol. 41, no. 7, pp. 27402779, 2012.

[12] R. Stiufiuc, C. Iacovita, C. M. Lucaciu et al., "SERS-active silver colloids prepared by reduction of silver nitrate with shortchain polyethylene glycol," Nanoscale Research Letters, vol. 8, no. 1, p. 47, 2013.

[13] S. K. Seol, D. Kim, S. Jung, W. S. Chang, and J. T. Kim, "Onestep synthesis of PEG-coated gold nanoparticles by rapid microwave heating," Journal of Nanomaterials, vol. 2013, Article ID 531760, 6 pages, 2013.

[14] J. A. Peck, C. D. Tait, B. I. Swanson, and G. E. Brown Jr, "Speciation of aqueous gold (III) chlorides from ultraviolet/visible absorption and Raman/resonance Raman spectroscopies," Geochimica et Cosmochimica Acta, vol. 55, no. 3, pp. 671676, 1991.

[15] I. Ojea-Jiménez and J. M. Campanera, "Molecular modeling of the reduction mechanism in the citrate-mediated synthesis of gold nanoparticles," The Journal of Physical Chemistry C, vol. 116, no. 44, pp. 23682-23691, 2012.
[16] F. Farges, J. A. Sharps, and G. E. Brown Jr, "Local environment around gold (III) in aqueous chloride solutions: an EXAFS spectroscopy study," Geochimica et Cosmochimica Acta, vol. 57, no. 6, pp. 1243-1252, 1993.

[17] P. Vanysek, "Electrochemical series," in CRC Handbook of Chemistry and Physics, vol. 8, Taylor \& Francis Group, 1999.

[18] C. Cruje, C. Yang, J. Uertz, M. van Prooijen, and B. D. Chithrani, "Optimization of PEG coated nanoscale gold particles for enhanced radiation therapy," RSC Advances, vol. 5, no. 123, pp. 101525-101532, 2015. 


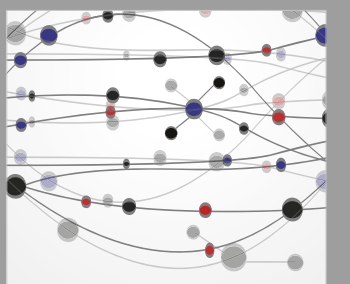

The Scientific World Journal
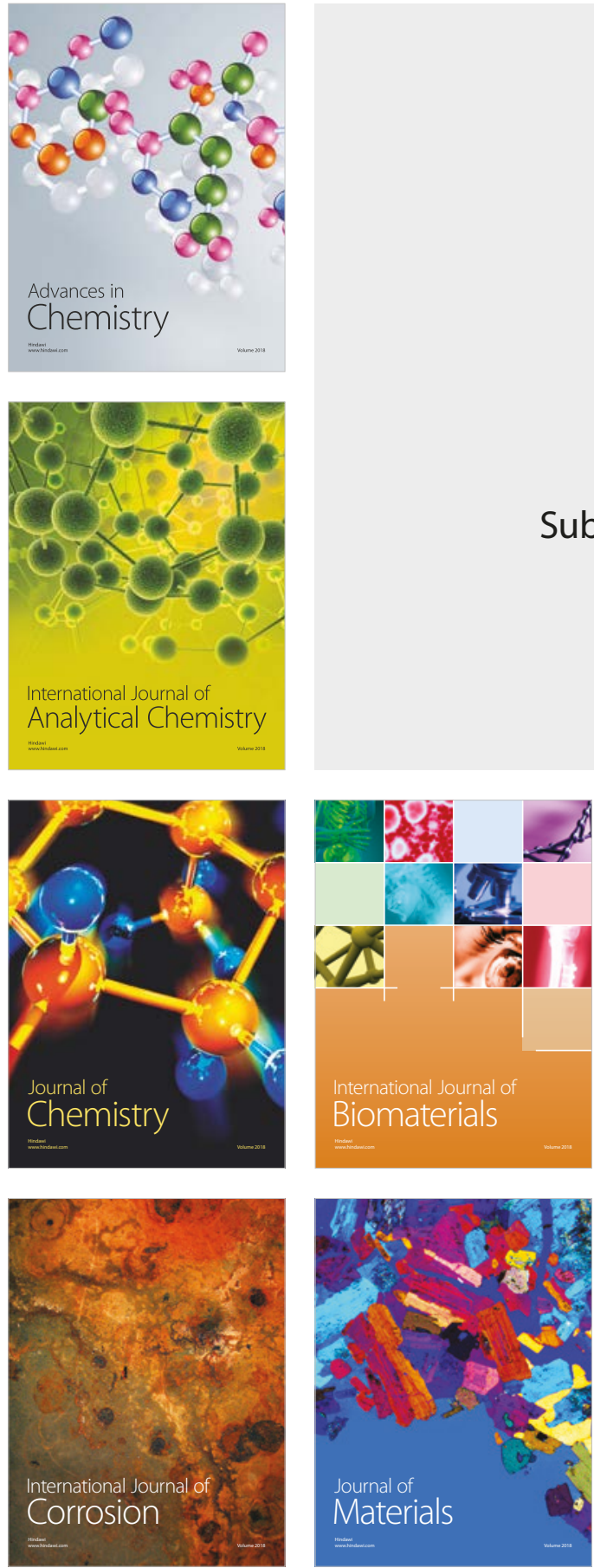

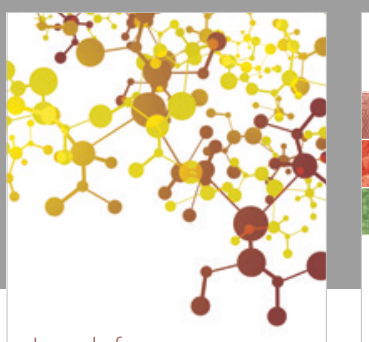

Journal of

Applied Chemistry
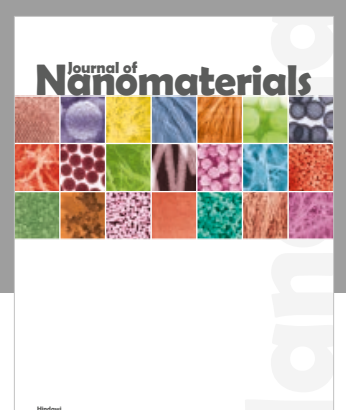

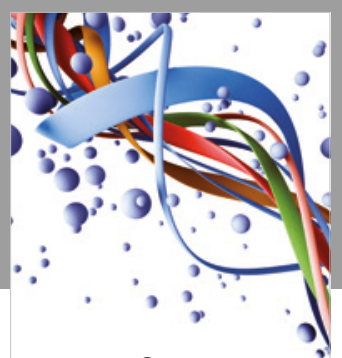

Scientifica

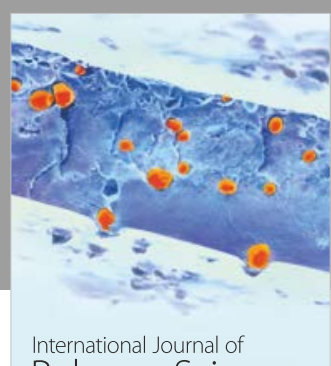

Polymer Science

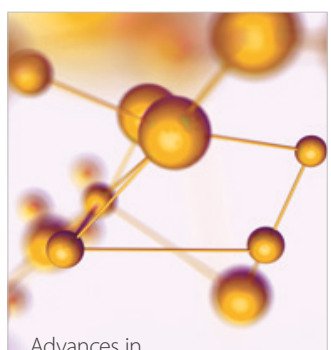

Physical Chemistry
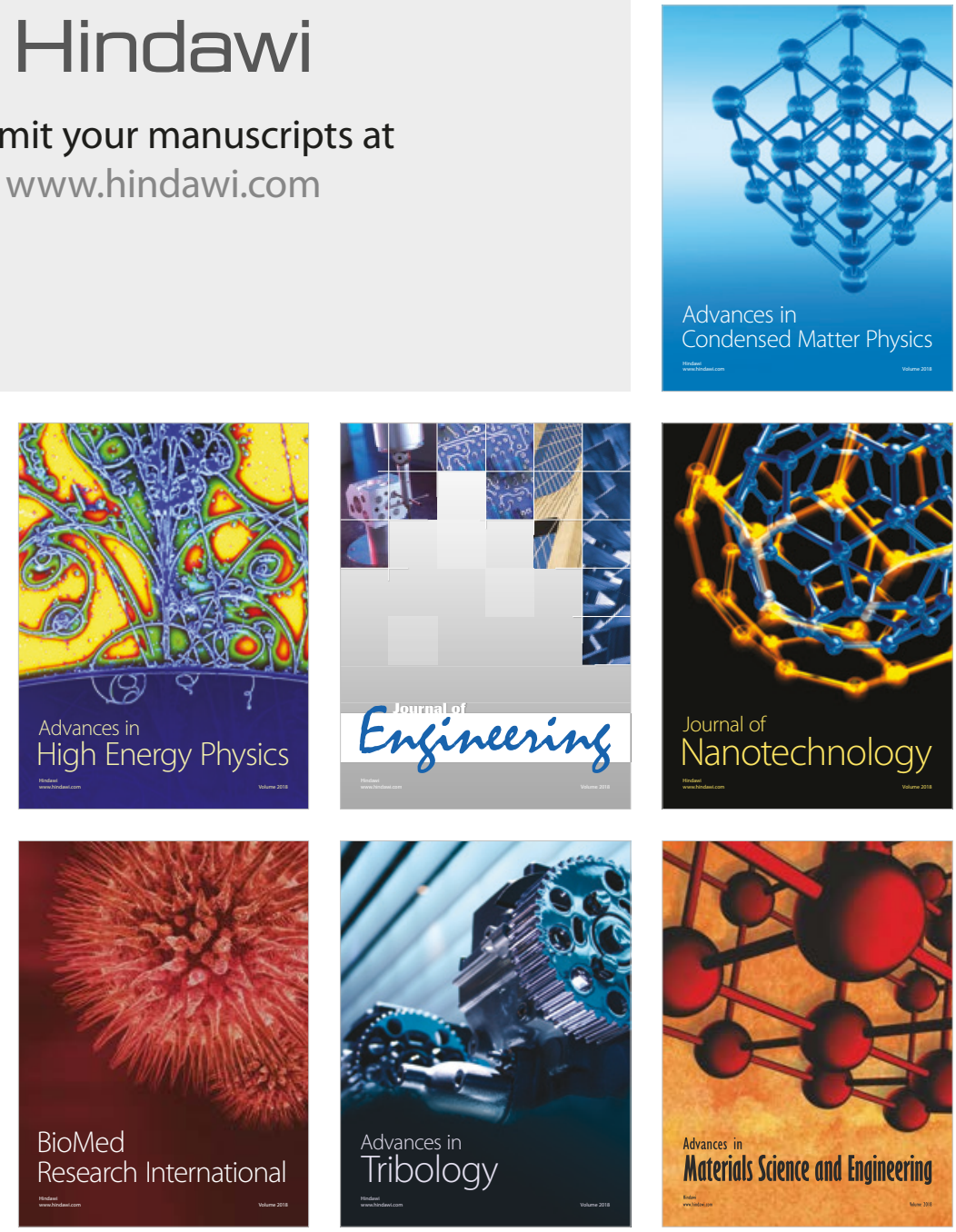